\title{
Rabi oscillations of two-photon states in nonlinear optical resonators
}

\author{
Y. Sherkunov, ${ }^{1, *}$ David M. Whittaker, ${ }^{2}$ and Vladimir Fal'ko ${ }^{1}$ \\ ${ }^{1}$ National Graphene Institute, University of Manchester, Manchester M13 9PL, United Kingdom \\ ${ }^{2}$ Department of Physics and Astronomy, University of Sheffield, Sheffield S3 7RH, United Kingdom
}

(Received 30 November 2015; published 26 February 2016)

\begin{abstract}
We demonstrate that four-wave mixing processes in high-quality nonlinear resonators can lead to Rabi-like oscillations in photon occupation numbers and second-order correlation functions, being a characteristic feature of the presence of entangled photon pairs in the optical signal. In the case of a system driven by a continuous coherent pump, the oscillations occur in the transient regime. We show that driving the system with pulsed coherent pumping would generate strongly antibunched photon states.
\end{abstract}

DOI: 10.1103/PhysRevA.93.023843

\section{INTRODUCTION AND MODEL}

Four-wave mixing (FWM) is a reversible process, in which a pair of photons, e.g., with a frequency $\omega_{0}$ is converted into two photons with frequencies $\omega_{1}$ and $\omega_{2}$, with $\omega_{1}+\omega_{2}=$ $2 \omega_{0}$. As a result, FWM could be used (instead of conventional down-conversion processes [1,2]) to create entangled photonpairs [3-7], suitable for practical implementation in quantum cryptography [8].

Here, we study FWM in high-quality resonators with discrete spectra [9] and strong Kerr nonlinearity: "zerodimensional" exciton-polariton microcavities [10-14] and microwave resonators coupled to superconducting qubits [15-18]. In these systems the FWM between photon pairs is reversible, so that quantum oscillations can occur between the initial and final states. We show that for a photon pair in a resonator, its states oscillate between two entangled two-photon configurations with the frequency determined by the strength of Kerr nonlinearity. For a pumped system, such Rabi-type oscillations would occur in transient regime after the pump is switched on, until losses damp the oscillations establishing a steady state. We suggest that such oscillations would be a characteristic feature of the presence of entangled photon pairs in the optical signal. We also demonstrate that applying pulsed driving to the system would generate photon states in the antibunching regime.

To model two-photon correlations in a nonlinear microcavity, we employ the master equation approach to the density matrix of the photon system, $\rho$, [19],

$$
\begin{aligned}
\frac{\partial \rho}{\partial t}= & -i\left[H+H_{p i}, \rho\right] \\
& +\gamma \sum_{i=0,1,2}\left(2 a_{i} \rho a_{i}^{\dagger}-a_{i}^{\dagger} a_{i} \rho-\rho a_{i}^{\dagger} a_{i}\right), \\
H= & \omega_{1} a_{1}^{\dagger} a_{1}+\omega_{2} a_{2}^{\dagger} a_{2}+\omega_{0} a_{0}^{\dagger} a_{0}+H_{n l}, \\
H_{n l}= & u a_{1}^{\dagger} a_{2}^{\dagger} a_{0}^{2}+\text { H.c.. }
\end{aligned}
$$

In (1), the Hamiltonian $H(\hbar=c=1)$ describes the conservative part of the photon system [20], $a_{i}$ and $a_{i}^{\dagger}$ are annihilation and creation operators of the photons in the mode $\omega_{i}, u$ is a photon-photon coupling constant due to Kerr nonlinearity,

*yury.sherkunov@manchester.ac.uk $\gamma$ is the photon decay rate, which is taken the same for all photon modes, and $H_{p i}$ describes the photon pump. Below, we consider microcavities excited using one of the two possible pumps sketched in Fig. 1,

$$
\begin{aligned}
& H_{p 1}=f(t)\left(e^{i \omega_{p 0} t} a_{0}+e^{-i \omega_{p 0} t} a_{0}^{\dagger}\right), \\
& H_{p 2}=f(t) \sum_{i=1,2}\left(e^{i \omega_{p i} t} a_{i}+e^{-i \omega_{p i} t} a_{i}^{\dagger}\right),
\end{aligned}
$$

where $f(t)$ and $\omega_{p i}$ are the amplitude and frequency of the pumping field. The pump (2a) excites photons in the mode $\omega_{0}$, while pump (2b) corresponds to coherent excitation of both $\omega_{1}$ and $\omega_{2}$ modes. By solving Eq. (1a) for the density matrix determined in the Fock space of states with different photon numbers, $m_{i}$ and $n_{i}$ in the three modes,

$$
\rho=\sum \rho\left(m_{1}, m_{2}, m_{0}, n_{1}, n_{2}, n_{0}\right)\left|m_{1}, m_{2}, m_{0}\right\rangle\left\langle n_{1}, n_{2}, n_{0}\right|,
$$

we evaluate the occupation numbers of photons and zero timedelay pair correlation functions for each mode $\omega_{i}$,

$$
\begin{gathered}
N_{i}=\operatorname{Tr} a_{i}^{\dagger} a_{i} \rho, \\
g_{i}^{(2)}=\operatorname{Tr}\left[\left(a_{i}^{\dagger}\right)^{2} a_{i}^{2}\right] / N_{i}^{2} .
\end{gathered}
$$

\section{RABI OSCILLATIONS OF TWO-PHOTON STATES IN CLOSED CAVITIES}

To introduce the idea of Rabi oscillations of two-photon states, let us consider an initial state of the system with two $\omega_{0}$ photons in an isolated $[f(t)=0]$ three-mode $\left(\omega_{0}, \omega_{1}, \omega_{2}\right)$ nondissipative $(\gamma=0)$ cavity, with $\delta=\omega_{1}+\omega_{2}-2 \omega_{0} \ll \omega_{i}$. The Hamiltonian of this system (1b) can be projected onto the reduced Fock space of the two-photon states, $\left\{\left|2_{\omega_{0}}\right\rangle,\left|1_{\omega_{1}}, 1_{\omega_{2}}\right\rangle\right\}$,

$$
H=\left(\begin{array}{cc}
2 \omega_{0} & \sqrt{2} u \\
\sqrt{2} u & \omega_{1}+\omega_{2}
\end{array}\right),
$$

with the eigenvalues and eigenstates,

$$
\begin{aligned}
& E_{ \pm}=\omega_{0}+\left(\omega_{1}+\omega_{2}\right) / 2 \pm \Omega / 2, \quad \Omega=\sqrt{\delta^{2}+8 u^{2}} \\
& \psi_{ \pm}=\frac{ \pm 1}{2 \sqrt{\Omega}}\left(\sqrt{\Omega \mp \delta}\left(a_{0}^{\dagger}\right)^{2} \pm \frac{4 u a_{1}^{\dagger} a_{2}^{\dagger}}{\sqrt{\Omega \mp \delta}}\right)|0\rangle .
\end{aligned}
$$

Here, $\Omega(\delta \rightarrow 0) \rightarrow 2 \sqrt{2} u$ is the two-photon Rabi frequency, which characterizes time-dependent oscillations of the 

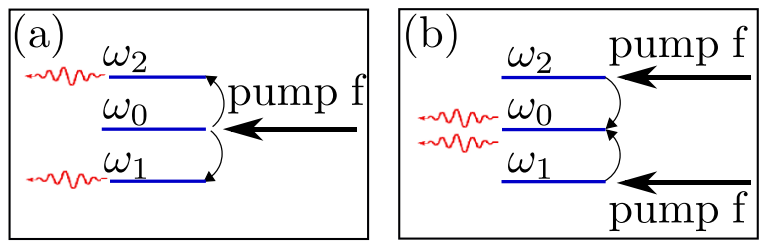

FIG. 1. Schematic diagram for a FWM process in a resonant microcavity with a discrete spectrum. (a) Coherent pump of the $\omega_{0}$ mode with subsequent scattering to the modes $\omega_{1}$ and $\omega_{2}$. (b) Coherent pump of two modes $\omega_{1}$ and $\omega_{2}$, generating a monochromatic pair in the $\omega_{0}$ mode.

probabilities $P\left(2_{\omega_{0}}\right)$ and $P\left(1_{\omega_{1}}, 1_{\omega_{2}}\right)$ to find these two photons,

$$
\begin{aligned}
P\left(2_{\omega_{0}}\right) & =\cos ^{2}\left(\frac{\Omega t}{2}\right)+\frac{\delta^{2}}{\Omega^{2}} \sin ^{2}\left(\frac{\Omega t}{2}\right), \\
P\left(1_{\omega_{1}}, 1_{\omega_{2}}\right) & =\frac{8 u^{2}}{\Omega^{2}} \sin ^{2}\left(\frac{\Omega t}{2}\right) .
\end{aligned}
$$

\section{RABI OSCILLATIONS IN OPEN RESONATORS}

In the case of a lossy system, with a finite decay rate $\gamma$, this result can be generalized into the formulas for probabilities to find two or one photon in the cavity, illustrated in Figs. 2(a) and 2(b),

$$
\begin{aligned}
P\left(2_{\omega_{0}}\right)= & e^{-4 \gamma t}\left[\cos ^{2}\left(\frac{\Omega t}{2}\right)+\frac{\delta^{2}}{\Omega^{2}} \sin ^{2}\left(\frac{\Omega t}{2}\right)\right], \\
P\left(1_{\omega_{1}}, 1_{\omega_{2}}\right)= & e^{-4 \gamma t} \frac{8 u^{2}}{\Omega^{2}} \sin ^{2}\left(\frac{\Omega t}{2}\right), \\
P\left(1_{\omega_{0}}\right)= & \frac{2 e^{-4 \gamma t}}{\Omega^{2}\left(\Omega^{2}+4 \gamma^{2}\right)} \\
& \times\left\{\left(e^{2 \gamma t}-1\right)\left(\Omega^{2}-4 u^{2}+4 \gamma^{2}\right) \Omega^{2}+16 \gamma u^{2}\right. \\
& \left.\times \sin \left(\frac{\Omega t}{2}\right)\left[\Omega \cos \left(\frac{\Omega t}{2}\right)+2 \gamma \sin \left(\frac{\Omega t}{2}\right)\right]\right\}, \\
P\left(1_{\omega_{1}}\right)= & P\left(1_{\omega_{2}}\right)=\frac{4 e^{-4 \gamma t} u^{2}}{\Omega^{2}\left(\Omega^{2}+4 \gamma^{2}\right)}\left\{\Omega^{2}\left(e^{2 \gamma t}-1\right)\right. \\
& -4 \gamma \sin \left(\frac{\Omega t}{2}\right)\left[\Omega \cos \left(\frac{\Omega t}{2}\right)\right. \\
& \left.\left.+2 \gamma \sin \left(\frac{\Omega t}{2}\right)\right]\right\} .
\end{aligned}
$$

These oscillations can manifest themselves in the temporal evolution of the occupation numbers $N_{i}$, illustrated in Fig. 2(c),

$$
\begin{aligned}
N_{0}= & \frac{2 e^{-4 \gamma t}}{\left(4 \gamma^{2}+\Omega^{2}\right) \Omega}\left\{e^{2 \gamma t} \Omega\left(4 \gamma^{2}+\Omega^{2}-4 u^{2}\right)\right. \\
& \left.+4 u^{2}[\Omega \cos (\Omega t)+2 \gamma \sin (\Omega t)]\right\}, \\
N_{1}= & N_{2}=\frac{4 u^{2} e^{-4 \gamma t}}{\left(4 \gamma^{2}+\Omega^{2}\right) \Omega}\left\{e^{2 \gamma t} \Omega\right. \\
& -[\Omega \cos (\Omega t)+2 \gamma \sin (\Omega t)]\} .
\end{aligned}
$$

In principle, the quantum-optical Rabi-type oscillations can occur in systems driven by coherent pumping. We demonstrate this for the case of resonant pumping, $\omega_{p i}=\omega_{i}$, and $\delta=0$. In the case of a lossless system $(\gamma=0)$ and weak continuous pumping switched on at $t=0, f(t)=($ const $\ll u) \times \theta(t)$, where $\theta(t)$ is the Heaviside function, Eq. (1a) can be solved analytically by diagonalizing the Hamiltonians (1b) and (2), which in the interaction representation can be written in the time-independent basis. This procedure leads to the following eigenvalues, $\{ \pm f, \pm \sqrt{2} u\}$ for pump (2a), and $\{0, \pm \sqrt{2} f, \pm \sqrt{2} u\}$ for pump (2b), and for $f t \ll 1$ results in the oscillations in occupation numbers $(\delta=0)$,

$$
N_{1}(t)=N_{2}(t)=\frac{f^{4} t^{2}}{u^{2}}\left(1-\frac{\sin (\Omega t / 2)}{\sqrt{2} u t}\right)^{2}
$$

for pump (2a), and

$$
N_{0}(t)=\frac{4 f^{4} t^{2}}{u^{2}}\left(1-\frac{\sin (\Omega t / 2)}{\sqrt{2} u t}\right)^{2}
$$

for pump (2b). Here, the sizable part of the oscillations has frequency $\Omega / 2$.

For a larger pumping amplitude, $f=($ const $\sim u) \times \theta(t)$, and for finite losses $\gamma \neq 0$, we solve Eq. (1a) numerically. We truncate the Fock space photon numbers, $m_{i}, n_{i} \leqslant N_{\max }$ and check that, for $f \sim u, N_{\max }=10$ is enough for the convergence (upon the increase of $N_{\max }$ ) of the numerical results. We find that photon pairs manifest themselves as Rabi-like oscillations between the two states $\left|2_{\omega_{0}}\right\rangle$ and $\left|1_{\omega_{1}}, 1_{\omega_{2}}\right\rangle$ as we show in Fig. 3 for the pumping scheme of Fig. 1(b). Interestingly, the oscillations of the occupation numbers can be well described by the two harmonics with frequencies close to $\Omega$ and $\Omega / 2$, as in the case discussed above, and can be fitted by the interpolation formula
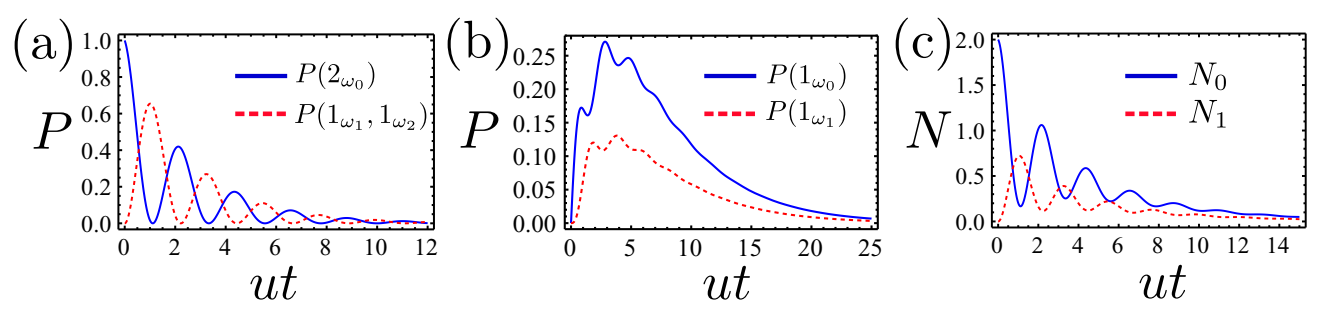

FIG. 2. Evolution of a two-photon state prepared in the mode $\omega_{0}$ in the resonator with damping $\gamma=0.1 u, \delta=0$, and $f=0$. (a) Probability distribution of two-photon states. (b) Probability distribution of single-photon states. (c) Average number of photons in the modes $\omega_{0}$ and $\omega_{1}$, or $\omega_{2}$. 

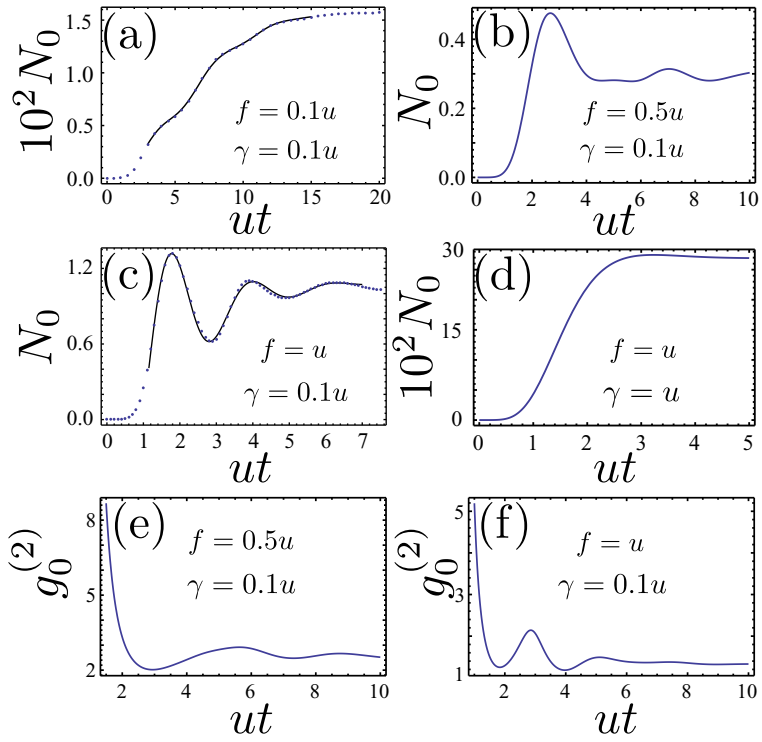

FIG. 3. Numerical results for occupation numbers in the mode $\omega_{0}, N_{0}(t)$, and two-photon correlation function for the mode $\omega_{0}, g_{0}^{(2)}$, in the continuously driven system sketched in Fig. 1(b). In (a) and (c), dots represent numerical results fitted by Eq. (11) (solid line). The fitting parameters are (a) $b_{1}=0.383, \alpha_{1}=3 \gamma, \phi_{1}=1.361$, and $b_{2}=0 ;$ (c) $b_{1}=0, b_{2}=3.023, \alpha_{2}=8 \gamma$, and $\phi_{2}=1.137$.

[Figs. 3(a), 3(c)],

$$
\begin{aligned}
N_{0}= & F(t)\left[1+b_{1} e^{-\alpha_{1} t} \cos \left(\frac{\Omega t}{2}+\phi_{1}\right)\right. \\
& \left.+b_{2} e^{-\alpha_{2} t} \cos \left(\Omega t+\phi_{2}\right)\right]
\end{aligned}
$$

where $F(t)$ is a monotonous (nonoscillating) function. For weak pumping, $f \ll u$, the amplitude of the $\Omega / 2$ harmonic, $b_{1}$, is much larger than the amplitude of the $\Omega$ harmonic, $b_{2}$, which is noticeable in Fig. 3(a). As the pumping amplitude $f$ increases, $b_{2}$ goes up and exceeds $b_{1}$ at $f \sim u$. Thus, the oscillations at $f \geqslant u$ have frequency $\Omega$ [Figs. 3(b)-3(c)]. The oscillations with the same frequencies can also be observed in the two-photon correlation function $g_{0}^{(2)}$ [Figs. 3(e)-3(f)].

We find a similar behavior for the pumping scheme illustrated in Fig. 1(a). In Figs. 4(a)-4(d), the oscillation frequency changes from $\Omega / 2$ for $f=($ const $\ll u) \times \theta(t)$ to $\Omega$ for $f=($ const $\sim u) \times \theta(t)$. There are also oscillations in the two-photon correlation function in the mode $\omega_{1}, g_{1}^{(2)}$, with the period matching the one of $N_{1}$. Note that, during its cycle, $g_{1}^{(2)}$ crosses the unit value, indicating switching from bunching to antibunching. We note that antibunching can be promoted, if the system is driven by pulses with a pump of finite duration $\tau$ corresponding to the minimum of $g_{1}^{(2)}$ in a continuously pumped system. As we show in Fig. 5 for the cavity driven by $\omega_{0}$-mode pulses, $f(t)=f_{0} \theta(\tau-t) \theta(t), g_{1}^{(2)}$ has a maximum corresponding to the bunching regime at the time when $N_{1}$ is small, but then, the system switches into the antibunching regime. In principle, the form of the ringing tones in oscillations of $N_{1}$ and $g_{1}^{(2)}$ may depend on the extent and shape of the excitation pulse; however this dependence is
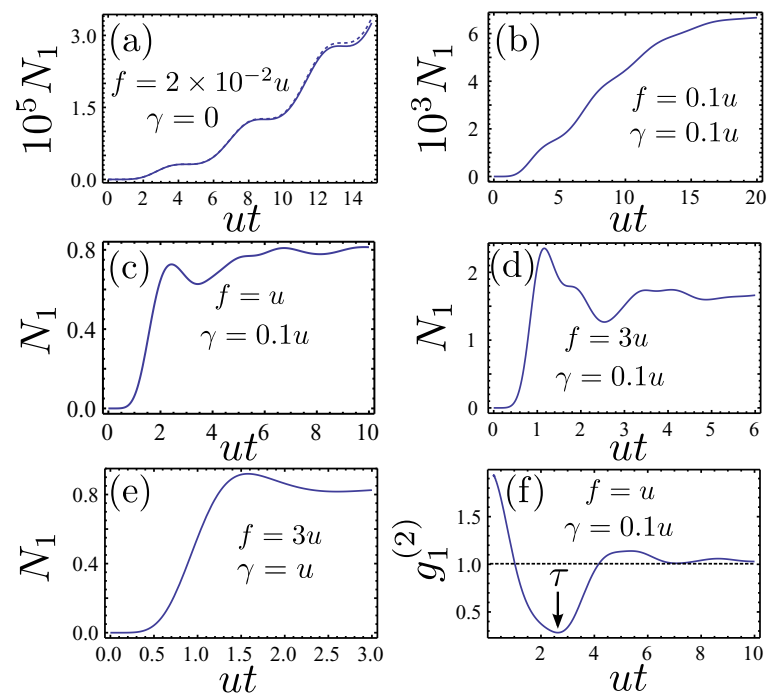

FIG. 4. Numerically evaluated occupation number of photons in the mode $\omega_{1}, N_{1}(t)$, and two-photon correlation function in the mode $\omega_{1}, g_{1}^{(2)}$, for the continuously driven system shown in Fig. 1(a). In (f), the arrow indicates the first minimum of $g_{1}^{(2)}$ at $\tau \approx 2.6 / u$. In (a), we compare the results of numerical simulations (solid line) and Eq. (9) (dashed line).

weak, as we show in Fig. 5, where we compare the systems excited by the pulses of various shapes.

\section{DISCUSSION}

In this paper, we have demonstrated that in nonlinear resonators with a discrete spectrum, correlated photon pairs can manifest themselves as quantum Rabi-like oscillations with the period determined by the strength of the nonlinearity. In coherently driven systems, these oscillations would occur in the transient regime, $t<1 / \gamma$, as shown in Figs. 3 and 4; thus, they could be observed in a system with sufficiently low damping, $\gamma \leqslant u$. To mention, the proposed theory describes systems with various frequency ranges. It can be applied to the visiblerange polaritonic microcavities based on GaAs, where the nonlinearity is due to exciton-exciton interaction in GaAs [21]. In these systems, the strength of nonlinearity, $u \sim 10 \mu \mathrm{eV}$, is still too weak as compared to damping, $\gamma \sim 80 \mu \mathrm{eV}$ [13]. However, we suggest that the use of two-dimensional transition metal dichalcogenides [22-24] in microcavities may improve
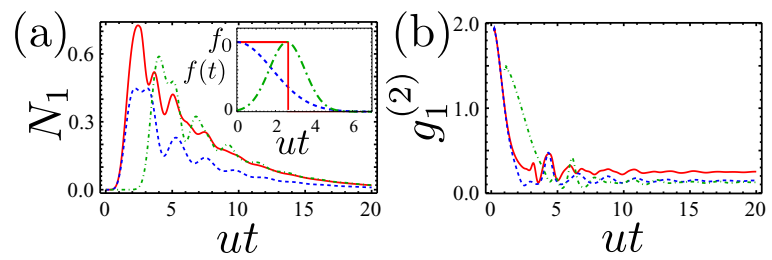

FIG. 5. Numerically evaluated $N_{1}(t)$ and $g_{1}^{(2)}$ for the system of Fig. 1(a) with $\gamma=0.1 u$ driven by the pulses (see inset): $f(t)=$ $f_{0} \theta(t) \theta(\tau-t)$ (red solid line); $f(t)=f_{0} \theta(t) \exp \left(-t^{2} / \tau^{2}\right)$ (dashed blue line); and $f(t)=f_{0} \exp \left[-4(t-\tau)^{2} / \tau^{2}\right]$ (green dot-dashed line) with amplitude $f_{0}=u$ and $\tau=2.6 / u$, corresponding to the minimum of $g_{1}^{(2)}$ of a continuously pumped system [Fig. 4(f)]. 
the $u / \gamma$ ratio due to stronger exciton-exciton interaction. Moreover, the proposed theory of two-photon oscillations is also applicable to microwave photons in superconducting resonators, where one can achieve quality factors in the range $10^{6}-10^{9}$ [25-27], hence reach the regime $u \gg \gamma$.

A large $u / \gamma$ ratio can also be achieved by resonantly coupling atoms with a level structure allowing for electromagnetically induced transparency (EIT) [28,29] and microcavities with high quality factors $Q$, such as toroidal $\left(Q>10^{8}\right)$ [30] or microrod $\left(Q>10^{9}\right) \quad$ [31] resonators. In these systems, the strength of the nonlinearity can reach $u \sim 1.25 \times 10^{7} \mathrm{~s}^{-1}$, while the losses can be as low as $\gamma \sim 2 \times 10^{5} \mathrm{~s}^{-1}[29,30,32,33]$, hence reaching the desirable regime $u \gg \gamma$ leading, as we show in Fig. 6, to Rabi oscillations in photon occupation numbers and the two-photon correlation function.

We have also shown that application of pulsed pumping to the system could lead to the generation of squeezed states of strongly antibunched photons.
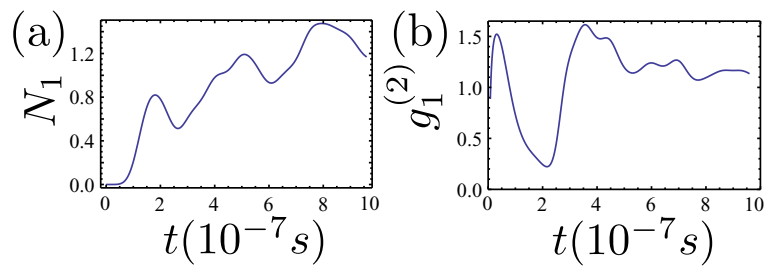

FIG. 6. Numerically evaluated (a) $N_{1}(t)$ and (b) $g_{1}^{(2)}$ for a highquality toroidal microcavity coupled to atoms in an EIT regime driven by resonant coherent pumping of Fig. 1(a) for an experimentally achievable set of parameters: $u=1.25 \times 10^{7} \mathrm{~s}^{-1}, \gamma=2 \times 10^{5} \mathrm{~s}^{-1}$, and $f=1.25 \times 10^{7} \mathrm{~s}^{-1}[29,30,32,33]$.

\section{ACKNOWLEDGMENTS}

We thank D. Krizhanovskii, E. Cancellieri, and M. Skolnick for useful discussions. This work was supported by EPSRC Programme Grant No. EP/J007544.
[1] P. G. Kwiat, K. Mattle, H. Weinfurter, A. Zeilinger, A. V. Sergienko, and Y. Shih, Phys. Rev. Lett. 75, 4337 (1995).

[2] I. Carusotto and G. C. La Rocca, Phys. Rev. B 60, 4907 (1999).

[3] L. J. Wang, C. K. Hong, and S. R. Friberg, J. Opt. B 3, 346 (2001).

[4] X. Li, P. L. Voss, J. E. Sharping, and P. Kumar, Phys. Rev. Lett. 94, 053601 (2005).

[5] J. Fulconis, O. Alibart, W. J. Wadsworth, and J. G. Rarity, New J. Phys. 9, 276 (2007).

[6] O. Cohen, J. S. Lundeen, B. J. Smith, G. Puentes, P. J. Mosley, and I. A. Walmsley, Phys. Rev. Lett. 102, 123603 (2009).

[7] R. Kumar, J. R. Ong, M. Savanier, and S. Mookherjea, Nat. Commun. 5, 5489 (2014).

[8] N. Gisin and R. Thew, Nat. Photon. 1, 165 (2007).

[9] By now, the studies of FWM processes have been focused on quasi-one-dimensional systems, such as optical fibres [34].

[10] O. El Daif, A. Baas, T. Guillet, J.-P. Brantut, R. I. Kaitouni, J. L. Staehli, F. Morier-Genoud, and B. Deveaud, Appl. Phys. Lett. 88, 061105 (2006).

[11] L. Ferrier, E. Wertz, R. Johne, D. D. Solnyshkov, P. Senellart, I. Sagnes, A. Lemaître, G. Malpuech, and J. Bloch, Phys. Rev. Lett. 106, 126401 (2011).

[12] S. Azzini, D. Gerace, M. Galli, I. Sagnes, R. Braive, A. Lemaitre, J. Bloch, and D. Bajoni, Appl. Phys. Lett. 99, 111106 (2011).

[13] S. Dufferwiel, F. Fras, A. Trichet, P. M. Walker, F. Li, L. Giriunas, M. N. Makhonin, L. R. Wilson, J. M. Smith, E. Clarke et al., Appl. Phys. Lett. 104, 192107 (2014).

[14] B. Besga, C. Vaneph, J. Reichel, J. Estève, A. Reinhard, J. Miguel-Sánchez, A. Imamoğlu, and T. Volz, Phys. Rev. Appl. 3, 014008 (2015).

[15] A. Wallraff, D. I. Schuster, A. Blais, L. Frunzio, R.-S. Huang, J. Majer, S. Kumar, S. M. Girvin, and R. J. Schoelkopf, Nature (London) 431, 162 (2004).

[16] L. S. Bishop, J. M. Chow, J. Koch, A. A. Houck, M. H. Devoret, E. Thuneberg, S. M. Girvin, and R. J. Schoelkopf, Nat. Phys. 5, 105 (2009).
[17] M. H. Devoret and R. J. Schoelkopf, Science 339, 1169 (2013).

[18] R. Barends, J. Kelly, A. Megrant, A. Veitia, D. Sank, E. Jeffrey, T. C. White, J. Mutus, A. G. Fowler, B. Campbell et al., Nature (London) 508, 500 (2014).

[19] Y. Sherkunov, D. M. Whittaker, H. Schomerus, and V. Fal'ko, Phys. Rev. A 90, 033845 (2014).

[20] In our analysis we neglect the effects of self- and cross phase modulation, which occur in a system with Kerr nonlinearity [35], although, we do not expect that these will affect the frequency of the oscillations but may enhance damping.

[21] V. Timofeev and D. Sanvitto, Exciton Polaritons in Microcavities (Springer, Berlin, 2012).

[22] L. Britnell, R. M. Ribeiro, A. Eckmann, R. Jalil, B. D. Belle, A. Mishchenko, Y.-J. Kim, R. V. Gorbachev, T. Georgiou, S. V. Morozov et al., Science 340, 1311 (2013).

[23] M. Palummo, M. Bernardi, and J. C. Grossman, Nano Lett. 15, 2794 (2015).

[24] S. Schwarz, S. Dufferwiel, P. M. Walker, F. Withers, A. A. P. Trichet, M. Sich, F. Li, E. A. Chekhovich, D. N. Borisenko, N. N. Kolesnikov et al., Nano Lett. 14, 7003 (2014).

[25] R. Barends, N. Vercruyssen, A. Endo, P. J. de Visser, T. Zijlstra, T. M. Klapwijk, P. Diener, S. J. C. Yates, and J. J. A. Baselmans, Appl. Phys. Lett. 97, 023508 (2010).

[26] K. Geerlings, S. Shankar, E. Edwards, L. Frunzio, R. J. Schoelkopf, and M. H. Devoret, Appl. Phys. Lett. 100, 192601 (2012).

[27] M. Reagor, H. Paik, G. Catelani, L. Sun, C. Axline, E. Holland, I. M. Pop, N. A. Masluk, T. Brecht, L. Frunzio et al., Appl. Phys. Lett. 102, 192604 (2013).

[28] A. Imamoḡlu, H. Schmidt, G. Woods, and M. Deutsch, Phys. Rev. Lett. 79, 1467 (1997).

[29] M. J. Hartmann and M. B. Plenio, Phys. Rev. Lett. 99, 103601 (2007).

[30] D. K. Armani, T. J. Kippenberg, S. M. Spillane, and K. J. Vahala, Nature (London) 421, 925 (2003).

[31] P. Del'Haye, S. A. Diddams, and S. B. Papp, Appl. Phys. Lett. 102, 221119 (2013). 
[32] T. Aoki, B. Dayan, E. Wilcut, W. P. Bowen, A. S. Parkins, T. J. Kippenberg, K. J. Vahala, and H. J. Kimble, Nature (London) 443, 671 (2006).

[33] S. M. Spillane, T. J. Kippenberg, K. J. Vahala, K. W. Goh, E. Wilcut, and H. J. Kimble, Phys. Rev. A 71, 013817 (2005).
[34] R. Z. Vered, Y. Shaked, Y. Ben-Or, M. Rosenbluh, and A. Pe'er, Phys. Rev. Lett. 114, 063902 (2015).

[35] L. Mandel and E. Wolf, Optical Coherence and Quantum Optics (Cambridge University Press, New York, 1995). 